\title{
Preclinical evaluation of a clinical prototype transrectal diffuse optical tomography system for monitoring photothermal therapy of focal prostate cancer
}

\author{
Celina L. Li, ${ }^{\text {a }}$ Carl J. Fisher $\odot,{ }^{\text {b }}$ Brian C. Wilson $\odot,{ }^{a, b}$ \\ and Robert A. Weersink $\oplus^{\text {a,b,c,d }}$ \\ ${ }^{a}$ University of Toronto, Department of Medical Biophysics, Toronto, Canada \\ ${ }^{b}$ University Health Network, Princess Margaret Cancer Centre, Toronto, Canada \\ ${ }^{\mathrm{c}}$ University of Toronto, Department of Radiation Oncology, Toronto, Canada \\ ${ }^{\mathrm{d} U n i v e r s i t y}$ of Toronto, Institute of Biomedical Engineering, Toronto, Canada
}

\begin{abstract}
Significance: Our work demonstrates in preclinical models that continuous-wave transrectal diffuse optical tomography (TRDOT) can be used to accurately monitor photothermal therapy (PTT) and, in particular, the progression of the photocoagulation boundary toward the rectum. When used in patients, this should prevent rectal damage during PTT, thereby achieving maximum treatment efficacy while ensuring safety, using a technology platform suitable for wide dissemination.
\end{abstract}

Aim: We aim to validate that TRDOT measurements analyzed using a shape-based imagereconstruction algorithm (SBDOT) allow localization of the photocoagulation boundary during PTT within $\pm 1 \mathrm{~mm}$ toward the rectum in the transverse plane.

Approach: TRDOT measurements were performed in tissue-simulating phantoms, ex vivo tissues, and an in vivo canine prostate model. The accuracy and sensitivity of reconstructing the size and location of the coagulation zone were determined, based on changes in the tissue absorption and reduced scattering coefficients upon photocoagulation. The reconstruction also yields the native and coagulated tissue optical properties.

Results: The TRDOT measurements and SBDOT reconstruction algorithm were confirmed to perform sufficiently well for clinical translation in PTT monitoring, recovering the location of the coagulation boundary within $\pm 1 \mathrm{~mm}$ compared to the true value as determined by direct visualization postexcision and/or MRI.

Conclusions: Implementing previously described TRDOT instrumentation and SBDOT image reconstruction in different tissue models confirms the potential for clinincal translation, including required refinements of the system and reconstruction algorithm.

(C) The Authors. Published by SPIE under a Creative Commons Attribution 4.0 International License. Distribution or reproduction of this work in whole or in part requires full attribution of the original publication, including its DOI. [DOI: 10.1117/1.JBO.27.2.026001]

Keywords: transrectal diffuse optical tomography; prostate cancer; photothermal therapy; image reconstruction; treatment monitoring.

Paper 210262RR received Aug. 18, 2021; accepted for publication Jan. 5, 2022; published online Feb. 1, 2022.

\section{Introduction}

Screening programs for prostate cancer (PCa) have increased the detection rate of low- and intermediate-risk disease confined to the prostate (focal tumors), for which treatment options range from active surveillance, with risk of disease progression, to radical therapies (prostatectomy and radiotherapy) that carry significant rates of urinary incontinence and erectile dysfunction. ${ }^{1,2}$ Focal ablative therapies, in which treatment is targeted to the dominant (index) lesion rather

*Address all correspondence to Robert A. Weersink, robert.weersink@rmp.uhn.ca 
than the whole gland, aim to minimize morbidity while achieving successful cancer control and preserving the possibility of retreatment. Several focal modalities, including high-intensity focused ultrasound, cryotherapy, irreversible electroporation, and photodynamic therapy, have shown promising results with short- to medium-term follow up. ${ }^{3-8}$

A further option is photothermal therapy (PTT), in which near-infrared (NIR) light is delivered via interstitial optical fibers to destroy the target index lesion by thermal coagulation $\left(>\sim 55^{\circ} \mathrm{C}\right){ }^{9-12}$ The advantages include good spatial control from accurate fiber insertion and selective tumor destruction regulated by light distribution depth and delivered energy. Photothermal treatment of focal PCa is usually monitored by magnetic resonance image-based thermometry (MRT), providing online feedback of thermal dose as an indirect indicator of the growth of the coagulation zone. Several clinical trials and case studies in low/intermediate-risk PCa patients have demonstrated that PTT can achieve minimal adverse effects and biopsyconfirmed tumor ablation in most cases, with $70 \%$ to $80 \%$ being radiologically and pathologically disease-free in the targeted ablation zone at 3 to 18 months follow up. ${ }^{13-18}$ However, due to the limited spatial resolution and indirect monitoring of the coagulation zone based on MRI temperature mapping, the photocoagulation front (boundary of thermal coagulation) cannot be delineated precisely enough to achieve complete tumor destruction in all cases, particularly for tumors in the posterior capsule, because of the need to prevent damage to the rectal wall. In one recent study, for example, only 11 out of 23 patients had complete target ablation. ${ }^{19}$

Diffuse optical tomography (DOT) uses visible/NIR light to sample a tissue volume of interest, allowing quantitative mapping of the optical absorption $\left(\mu_{a}\right)$ and reduced scattering $\left(\mu_{s}^{\prime}\right)$ coefficients that carry morphological and functional information. ${ }^{20}$ DOT can be used stand alone or in combination with radiological imaging. ${ }^{21}$ Transrectal DOT (TRDOT) has also been investigated for prostate tumor imaging with transrectal ultrasound (TRUS) to enhance spatially accuracy. ${ }^{22-25}$ We have previously reported the design, construction, and calibration of a prototype continuous-wave DOT system and transrectal probe. Preliminary tests demonstrated that TRDOT signals are sensitive to changes in tissue absorption and scattering due to PTT ${ }^{26-28}$ and had sufficient dynamic range. The probe is shown in Fig. 1.

We also previously reported a shape-based algorithm (SBDOT) to reconstruct images of the treatment zone, ${ }^{29}$ based on assuming an approximately ellipsoidal, sharp, and monotonically expanding boundary between the coagulated and native tissue. Computer simulations ${ }^{26}$ and preliminary phantom experiments ${ }^{27,28}$ suggested that it is possible to localize the coagulation boundary within $\pm 1 \mathrm{~mm}$ in the transverse plane, which we had set as the target accuracy to avoid rectal damage while allowing maximal tumor ablation. We demonstrated that a twofold increase in scattering upon coagulation is required for reconstruction accuracy. ${ }^{26,29}$

The primary objective of the current work is then to validate these prelimary findings systematically and in depth, including under in vivo conditions, as a critical step toward clinical translation. Hence, we first validated the SBDOT algorithm in tissue-simulating phantoms to

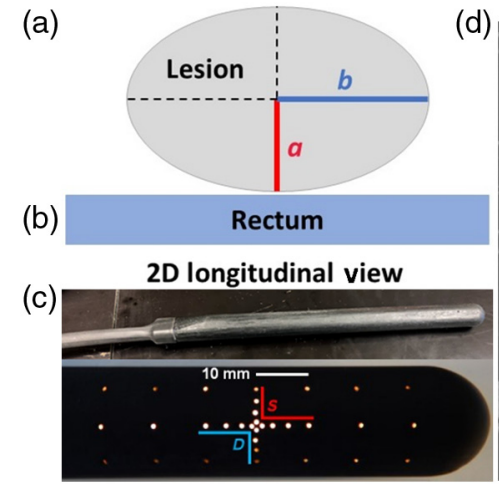

(d)

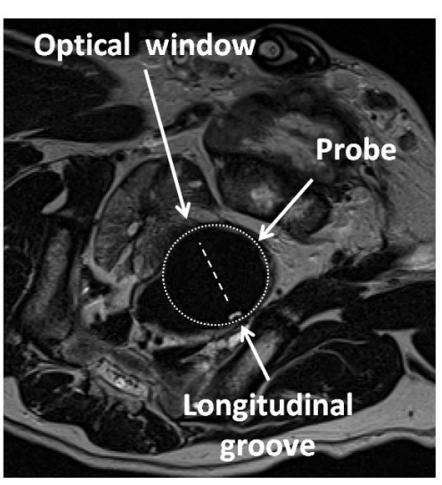

Fig. 1 Transrectal DOT probe: (a) longitudinal view showing the geometry of the photothermal coagulation zone relative to the rectum and indicating the transverse $(a)$ and longitudinal $(b)$ radii. (b) Photograph of the probe and (c) arrangement of source $(S)$ and detector $(D)$ fibers within the optical window. (d) MR image (transverse plane) of the probe in canine rectum: the longitudinal orientation groove is visible. 
reconstruct zones of fixed diameter and location and with known optical properties representative of coagulated tissue. DOT monitoring of the coagulation zone during PTT was then tested in ex vivo tissues under well-controlled conditions using a simulated transrectal geometry. This allowed detailed quantitative comparisons between the reconstructed photocoagulation zone compared to simultaneous MR thermometry as treatment progressed. Finally, the system and reconstructions were validated under clinically relevant in vivo conditions during photothermal treatment of normal prostate in a canine model with the TRDOT probe placed in the rectum. These studies critically inform planned first clinical trials of this treatment monitoring technology.

\section{Materials and Methods}

All canine procedures were approved by the Animal Care Committee, University Health Network, Toronto, Canada and complied with regulations of the Canadian Council on Animal Care.

\subsection{Instrumentation and Data Acquisition}

Details of the measurement and reconstruction workflow are described in the Supplementary Material. The system currently utilizes three wavelengths: 670,750, and $808 \mathrm{~nm}$ corresponding, respectively, to the main absorption peak of lipid-porphyrin nanoparticles under development for thermal dose enhancement during PTT, ${ }^{30}$ an absorption peak of deoxygenated hemoglobin $(\mathrm{Hb})$, and the $\mathrm{Hb}-\mathrm{HbO}_{2}$ isosbestic point. The MRI-compatible transrectal probe is cylindrical (diameter $25 \mathrm{~mm}$ ) with $400 \mu \mathrm{m}$ optical fibers arranged within an area $(20 \mathrm{~mm}$ longitudinally $\times 16 \mathrm{~mm}$ transversally) centred $5 \mathrm{~cm}$ from the probe tip. The eight light delivery and eight collection fibers exit the probe perpendicular to and flush with the surface, as seen in Fig. 1. Powers of 20, 9, and $5.5 \mathrm{~mW}$ were used for the 670-, 750-, and 808-nm lasers, respectively. Grooves in the longitudinal and the transversal planes enable MRI positioning and orientation. Phantom measurements were made at 670,750 , and $808 \mathrm{~nm}$, whereas ex vivo and in vivo measurements were made only at $750 \mathrm{~nm}$ to minimize the sampling time in following the dynamic coagulation changes: 28 s per scan using 8 sources when using 8 sources. Measurements in a reference phantom of known optical properties provide calibration factors for each source-detector pair, ${ }^{28}$ repeated for each measurement session.

\subsection{Phantom Study}

Phantom mixtures were prepared using laboratory-grade agar (BioShop Canada Inc., Burlington, Ontario, Canada) at $2 \%$ weight/volume as the bulk matrix, Intralipid ${ }^{\circledR} 20 \%$ stock solution (Baxter, Mississauga, Ontario, Canada) for optical scattering, India Ink for optical absorber, and 5\% w/v OMNIPAQUE 350 iohexol injection USP (GE Healthcare, Mississauga, Ontario, Canada) as a CT contrast agent and $0.8 \% \mathrm{NaCl}$ as an antibacterial agent. A previously validated spatially resolved flat-top diffuse reflectance (DR) probe was used to measure the optical properties ${ }^{31}$ in a 6-cm diameter, $2.5-\mathrm{cm}$ height phantom block. Separately, two cylindrical wells were fabricated in a $1000-\mathrm{ml}$ container, one of $20-\mathrm{mm}$ diameter representing the coagulated zone with altered optical properties and one of 25-mm diameter for the probe. Each well was 100-mm deep with an edge-to-edge separation of $2.7 \mathrm{~mm}$. The sizes and separation of the wells were confirmed by cone-beam CT imaging: Fig. 2(a). The 20 -mm well was filled to a height of $\sim 90 \mathrm{~mm}$ using six different concentrations of Intralipid and India Ink to represent zones of altered, i.e., coagulated, optical properties. The optical properties of each liquid inclusion and of the agar matrix were measured at 670, 750, and $808 \mathrm{~nm}$ using the DR probe that was independently calibrated in a commercial solid reference phantom (Biomimic ${ }^{\mathrm{TM}}$, INO, Quebec, Canada). ${ }^{28}$

The probe well was water filled for optical coupling. All measurements were repeated 5 times with the phantom covered with blackout fabric and were corrected for dark counts [Fig. 2(b)]. Reconstructions were performed using different initial estimates of the inclusion radius $(6,8,10$, and $12 \mathrm{~mm}$ ) and length $(10$ and $45 \mathrm{~mm})$ combined with different regularization values. Estimated errors in the reconstructed optical properties and shape parameters were calculated using the 


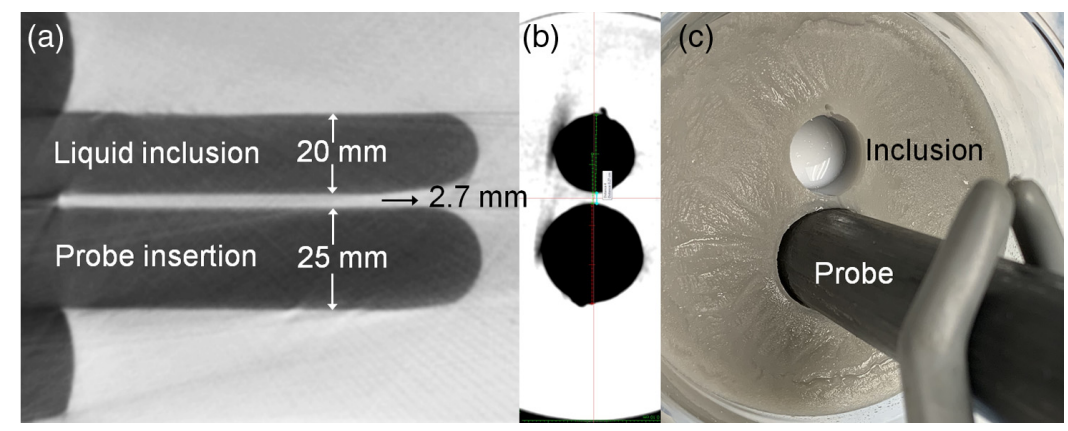

Fig. 2 Agar phantom with inclusion simulating the photocoagulation zone and holding the probe. Cone-beam CT images in (a) coronal and (b) axial views $\left(0.39 \times 0.39 \times 0.39 \mathrm{~mm}^{3}\right.$ voxels). (c) Top-view photograph.

standard methods for nonlinear regressions based on calculating the Hessian matrix. The optical properties of the simulated coagulation zone and the agar phantom as measured by the DR probe were used as initial values in reconstruction and we also examined the effect of varying the initial $\mu_{a}$ and $\mu_{s}^{\prime}$ values, both individually and in combination, using the methodology shown in detail in Fig. S2 in the Supplementary Material. The reconstructions were based on NIRFAST ${ }^{32}$ meshing and were performed in MATLAB (The MathWorks, Inc., Natick, Massachusetts, USA). CT images were analyzed using ImageJ (National Institutes of Health, Bethesda, Maryland, USA).

\subsection{Ex Vivo Study}

TRDOT monitoring of photocoagulation was performed in ex vivo bovine and porcine muscle using a setup similar to the prior preliminary experiments. ${ }^{28}$ Briefly, two 8 - to 12 -mm-thick tissue slabs of either bovine or porcine muscle were used. The probe was fixed with the optical window facing upward on the table of a 1.5-T MRI system (Aera: Siemens, Toronto, Ontario, Canada). A first tissue slab was placed on top in close contact. A 1.7-mm diameter, 15-mm long diffusing-tip PTT treatment fiber delivering $5 \mathrm{~W}$ at $980 \mathrm{~nm}$ was placed on the tissue parallel to the long axis of the probe and centered on the optical window. The fiber and probe positions were confirmed by a fast MRI localization scan. $\mathrm{T}_{2} \mathrm{w}$ Turbo Spin Echo scans then were acquired preand post-PTT to image the photocoagulation zone in the axial plane (voxel size: $1 \times 1 \times 3 \mathrm{~mm}^{3}$; TR: $3600 \mathrm{~ms}$; and TE: $57 \mathrm{~ms}$ ). Repeated MRT scans (GRE; TR: $46 \mathrm{~ms}$; TE: $9 \mathrm{~ms} ; 1 \times 1 \times$ $3 \mathrm{~mm}^{3}$ voxels) and $750 \mathrm{~nm}$ DOT scans were acquired simultaneously for $\sim 140 \mathrm{~s}$ immediately prior to PTT, $\sim 700 \mathrm{~s}$ during treatment delivery, and $\sim 300$ to $400 \mathrm{~s}$ during tissue cool down. The tissue slabs were then separated and the final photocoagulation diameter was measured using a ruler in the longitudinal and cross-sectional directions.

Rather than a reference phantom, the native tissue optical properties, either measured using the DR probe or from the literature were used for TRDOT calibration, to minimize any effect of tissue-probe contact. These properties were input to a forward model of the TRDOT signals, which were compared to the measured signals to calculate individual source-detector calibration factors. The transverse and longitudinal radii and the optical properties of the coagulation zone were reconstructed using the SBDOT algorithm. For comparison, following $3 \times 3$ Gaussian smoothing, the $55^{\circ} \mathrm{C}$ contour from the MRT images was taken as the photocoagulation boundary at each treatment time point, noting in particular the distance between the coagulation boundary and the probe. The PTT fiber position and final photocoagulation boundary were also estimated from the posttreatment T2w MR images. As detailed in the Supplementary Material S2, a range of initial optical properties of the coagulation tissue were used in the SBDOT reconstructions to test the sensitivity to these starting values.

\subsection{In Vivo Study}

In vivo PTT of the canine prostate, which is similar in size to the adult human prostate, was performed in 6 male beagle dogs (12- to 14-month old, 12 to $14 \mathrm{~kg}$ weight: Marshall 


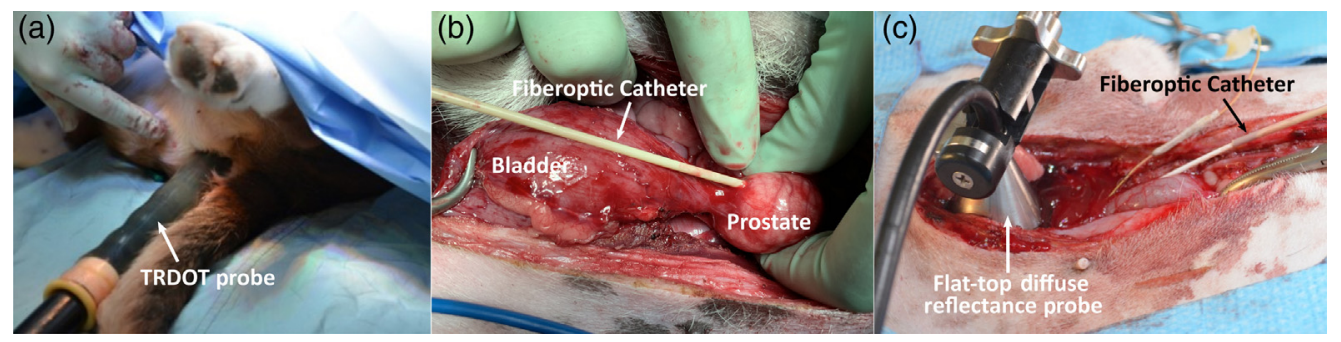

Fig. 3 In vivo setup showing (a) insertion of the treatment fiber at laparotomy; (b) the TRDOT probe placed in the rectum with the optical window facing the center of prostate; and (c) the flat-top DR probe held rigidly on the ventral surface of the exposed prostate.

Bioresources Inc., NY, USA). All procedures were performed under general anesthesia following $12 \mathrm{~h}$ fasting and 3 warm-water enemas. Laparotomy, as required for a different concurrent study, allowed direct access to the prostate. For the laparotomy, an incision was made from the umbilicus to the pubis, exposing the bladder, prostate, and kidney. The bladder was retracted toward the umbilicus and sutured to the abdominal wall to minimize free-organ motion, which also exposed and stabilized the prostate. The PTT treatment fiber was inserted through a 14-G cannula/introducer to a depth of $\sim 1 \mathrm{~cm}$ [see Fig. 3(a)].

The TRDOT probe was inserted into the rectum in a sterile condom with lubricant gel and aligned with the prostate [Fig. 3(b)] by palpation. The optical properties of the untreated prostate at $750 \mathrm{~nm}$ were measured using the DR probe in direct contact with the anterior prostate surface via the laparotomy [Fig. 3(c)]. All TRDOT scans were performed at $750 \mathrm{~nm}$, starting immediately prior to PTT. The first half of the treatment was given using a laser power of $3 \mathrm{~W}$, which was increased to $5 \mathrm{~W}$ at the midpoint. This protocol minimized blood coagulation at the fiber tip while maximizing the coagulation zone. It also tested the ability of TRDOT to track the growth rate of the coagulation zone. Two different TRDOT monitoring protocols were evaluated: (i) PTT over $744 \mathrm{~s}$, with TRDOT monitoring using 8 light sources ( $28 \mathrm{~s}$ per complete scan) and (ii) PTT for $210 \mathrm{~s}$ and TRDOT using only 4 sources to reduce the acquisition time to $14 \mathrm{~s}$. In both cases, TRDOT monitoring continued for $\sim 100 \mathrm{~s}$ following the end of treatment.

The SBDOT algorithm was used to reconstruct the expanding photocoagulation zone and to measure the pre- and post-PTT optical properties. This utilized a NIRFAST mesh comprising four regions: photocoagulation zone, untreated prostate, rectum, and surrounding tissues. TRDOT data were calibrated as per the ex vivo study.

\section{Results and Discussion}

\subsection{Phantom Study}

The $\mu_{a}$ and $\mu_{s}^{\prime}$ values of the agar phantom were 0.009 and $1.08 \mathrm{~mm}^{-1}, 0.006$ and $1.07 \mathrm{~mm}^{-1}$, and 0.004 and $1.01 \mathrm{~mm}^{-1}$ at 670,750 , and $808 \mathrm{~nm}$, respectively, with coefficients of variation of $9.8 \%$ for $\mu_{a}$ and $4.0 \%$ for $\mu_{s}^{\prime}(n=10)$. The optical properties of the liquid inclusions simulating the coagulation zone as calculated from the TRDOT measurements are plotted against the reference DR probe measurements in Fig. 4. The SBDOT reconstructed radius was $r=10.8 \pm 0.2 \mathrm{~mm}$ (where the error represents the average across measurements at the three wavelengths), compared with the true value of $10 \mathrm{~mm}$. Table 1 summarizes the average reconstructed values at each wavelength.

TRDOT recovered the scattering coefficients within $\pm 3 \%$ but systematically underestimated the absorption by about $20 \%$. The impact of errors in reconstructed optical properties on the reconstructed size was assessed by fixing the optical properties over a range from $-100 \%$ to $+300 \%$ of the true values, whereas the scattering coefficient was varied from $-100 \%$ to $200 \%$. Variations in $\mu_{a}$ of up to $\pm 30 \%$ and in $\mu_{s}^{\prime}$ of up to $\pm 40 \%$ caused $<0.7 \mathrm{~mm}$ variations in the reconstructed inclusion radius. The reconstructed longitudinal size converged to the maximum dimension $(20 \mathrm{~mm})$ of the optical window, which was shorter than the actual value of $45 \mathrm{~mm}$. 

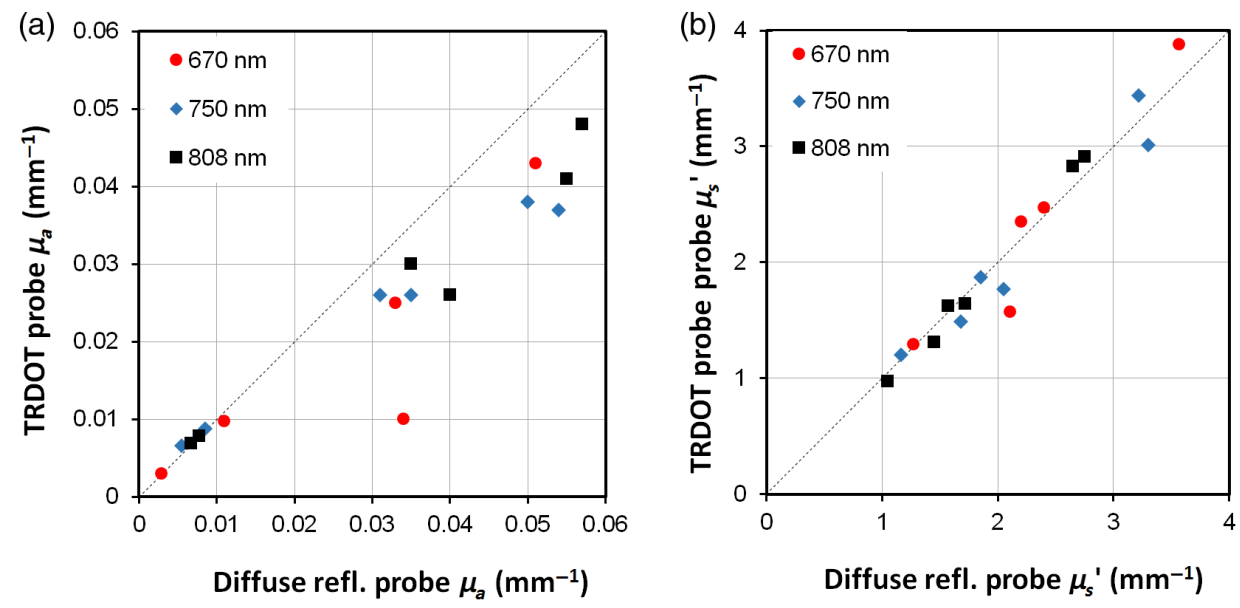

Fig. 4 Comparison of (a) $\mu_{a}$ and (b) $\mu_{s}^{\prime}$ of the liquid inclusions measured by the TRDOT and flat-top DR probes. The signal was below the measurement limit at $670 \mathrm{~nm}$ with the highest absorption. Lines of equality are indicated.

Table 1 Inclusion optical properties showing the target range of $\mu_{a}$ and $\mu_{s}^{\prime}$, the measured range over 670,750 , and $808 \mathrm{~nm}$ using the DR probe and the TRDOT probe.

\begin{tabular}{|c|c|c|c|c|c|c|}
\hline & \multicolumn{3}{|c|}{$\mu_{a}\left(\mathrm{~mm}^{-1}\right)$} & \multicolumn{3}{|c|}{$\mu_{s}^{\prime}\left(\mathrm{mm}^{-1}\right)$} \\
\hline & Target & DR & TRDOT & Target & DR & TRDOT \\
\hline Inclusion 1 & 0.007 to 0.010 & 0.007 to 0.011 & 0.008 to 0.01 & 1.05 to 1.30 & 1.05 to 1.27 & 1.0 to 1.3 \\
\hline Inclusion 2 & 0.007 to 0.010 & 0.003 to 0.007 & 0.003 to 0.007 & 1.85 to 2.40 & 1.7 to 2.4 & 1.64 to 2.47 \\
\hline Inclusion 3 & 0.03 to 0.036 & 0.034 to 0.04 & 0.01 to 0.026 & 1.85 to 2.40 & 1.45 to 2.1 & 1.31 to 1.57 \\
\hline Inclusion 4 & 0.05 to 0.06 & 0.051 to 0.055 & 0.038 to 0.043 & 1.85 to 2.40 & 1.6 to 2.2 & 1.62 to 2.35 \\
\hline Inclusion 5 & 0.03 to 0.036 & 0.031 to 0.035 & 0.025 to 0.03 & 2.75 to 3.50 & 2.7 to 3.6 & 2.83 to 3.88 \\
\hline Inclusion $6^{a}$ & 0.05 to 0.06 & 0.054 to 0.057 & 0.037 to 0.048 & 2.75 to 3.50 & 2.9 to 3.4 & 2.91 to 3.44 \\
\hline
\end{tabular}

${ }^{a}$ Measurements for inclusion 6 do not include data at $670 \mathrm{~nm}$ due to high-signal attenuation affecting recovery of optical properties.

However, this was as expected and the window is long enough to encompass most clinical cases, in which the diffusing optical fibers for treatment light delivery rarely exceed $30 \mathrm{~mm}$.

Generally, the reconstructions were insensitive to varying the initial input values for the optical properties and lesion size. For example, varying the initial estimate of the transverse lesion radius between 2 and $12 \mathrm{~mm}$ gave a coefficient of variation of only $\sim 0.2 \mathrm{~mm}$ in radius across 21 samples. When the initial optical properties were varied by $-40 \%$ to $+80 \%$ of the true values, the reconstructed radius was $10.5 \pm 0.6 \mathrm{~mm}$ averaged over all reconstructions $(n=99)$ from the 670-, 750-, and 808-nm data. Only when these initial estimates were varied by more than $+100 \%$ or $-40 \%$ did the reconstructed transverse radius deviate from the true values by more than $2 \mathrm{~mm}$ (Fig. S3 in the Supplementary Material). Published optical properties of human in vivo prostate range widely, ${ }^{33-36}$ from 0.01 to $0.16 \mathrm{~mm}^{-1}$ for $\mu_{a}$ and from 0.1 to $4 \mathrm{~mm}^{-1}$ for $\mu_{s}^{\prime}$ between 730 and $790 \mathrm{~nm}$, depending on measurement technique. The effective attenuation coefficient $\mu_{\text {eff }}$ is more consistent at between 0.20 and $0.33 \mathrm{~mm}^{-1}$, and within a patient group has a lower standard deviation than $\mu_{a}$ and $\mu_{s}^{\prime}{ }^{34}$ If the primary source of these variations is technical rather than biological, then the confidence interval for the starting optical properties should converge as the clinical data using TRDOT during PTT increases, thereby further improving reconstruction of the coagulation zone boundary. 


\subsection{Ex Vivo Study}

Table 2 summarizes the initial ("native") optical properties of each tissue used for the reconstructions as well as the coagulated tissues. Due to low absorption and scattering of porcine muscle, the native optical properties were not accurately recovered using the DR probe, so that the initial estimates were also extrapolated from literature values at $600 \mathrm{~nm}^{37}$ and $750 \mathrm{~nm} .^{38-43}$

For the coagulated tissues, the measured scattering coefficients for bovine muscle using either the DR or TRDOT probes are consistent with literature values, while those for porcine tissue are $\sim 50 \%$ of the literature value. The absorption coefficients vary markedly between the DR probe, TRDOT probe, and literature values. Most likely this reflects differences in blood content due to tissue collection and handling, as could be resolved by measuring at wavelengths above and below the $\mathrm{Hb}$ isosbestic point.

Figure 5 shows photographs of the coagulation zone, each at 15 min post-PTT, MRI $\mathrm{T}_{2} \mathrm{~W}$ images overlaid with an estimate of coagulation zone boundary based on contrast within each image, as well as MRT images taken immediately at the end of treatment, with the $55^{\circ} \mathrm{C}$ contour

Table 2 Optical properties of native and coagulated tissues at $750 \mathrm{~nm}$ measured with the TRDOT and fat-top DR probes, and compared to the literature values. ${ }^{37-43}$

\begin{tabular}{|c|c|c|c|c|c|c|}
\hline & \multirow{2}{*}{$\begin{array}{c}\text { Optical } \\
\text { property } \\
\left(\mathrm{mm}^{-1}\right)\end{array}$} & \multicolumn{2}{|c|}{ Native } & \multicolumn{3}{|c|}{ Coagulated } \\
\hline & & DR & Literature & TRDOT & DR & Literature \\
\hline \multirow[t]{2}{*}{ Porcine } & $\mu_{a}$ & & 0.01 to 0.025 & $0.072 \pm 0.007$ & $0.036 \pm 0.005$ & 0.10 \\
\hline & $\mu_{s}^{\prime}$ & & 0.3 to 0.4 & $1.4 \pm 0.4$ & $1.5 \pm 0.1$ & 3.0 \\
\hline \multirow[t]{2}{*}{ Bovine } & $\mu_{a}$ & $0.0040 \pm 0.0005$ & 0.01 to 0.03 & $0.062 \pm 0.005$ & $0.028 \pm 0.007$ & 0.03 to 0.04 \\
\hline & $\mu_{s}^{\prime}$ & $0.87 \pm 0.01$ & 0.34 to 0.70 & $2.3 \pm 0.3$ & $2.3 \pm 0.3$ & 1.5 to 2 \\
\hline
\end{tabular}

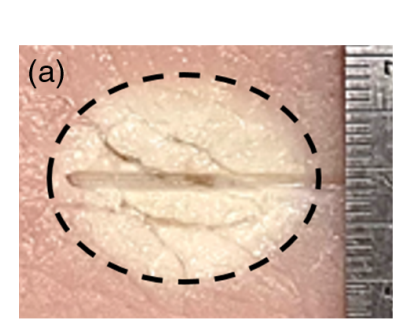

Porcine

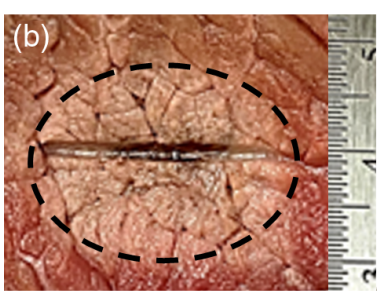

Bovine
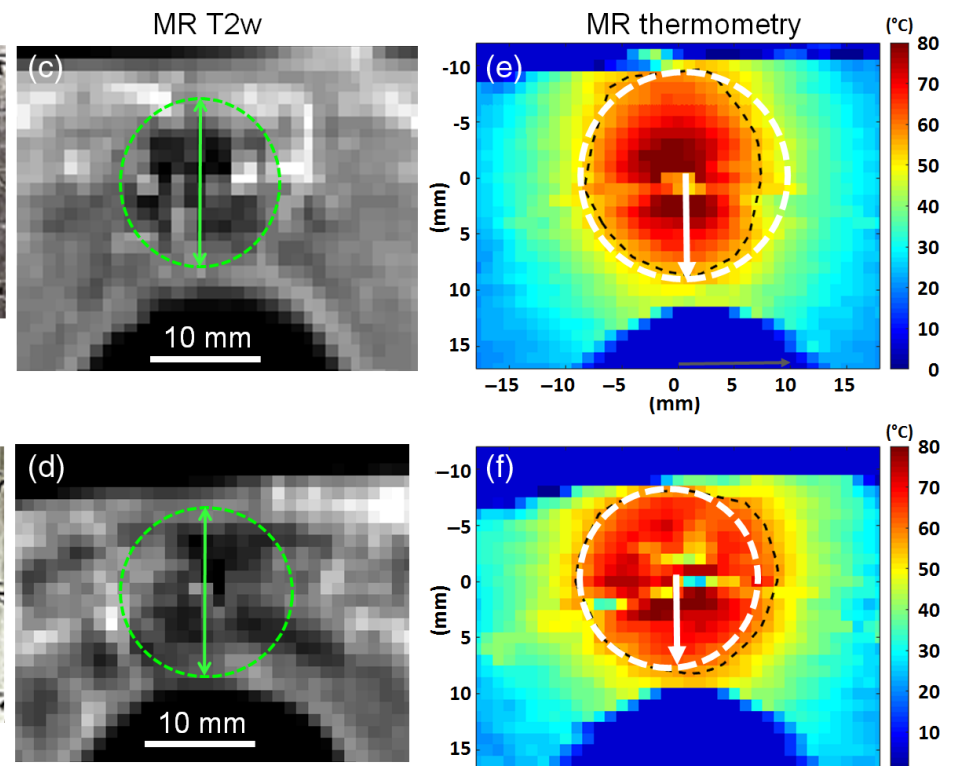

Fig. 5 Examples of post-PTT coagulation zones in ex vivo tissues: (top) porcine and (bottom) bovine. (a), (b) Photographs at $15 \mathrm{~min}$ post-PTT in the coronal plane with TRDOT boundary shown as dashed black lines. (c), (d) Transverse MR $\mathrm{T}_{2} \mathrm{w}$ images with the lesion boundary outlined in green. (e), (f) False-color MRT images showing the $55^{\circ} \mathrm{C}$ temperature contours (black dashed line) immediately at the end of PTT. White dashed circles represent the reconstructed TRDOT boundaries centered on the treatment fiber. 
Li et al.: Preclinical evaluation of a clinical prototype transrectal diffuse optical tomography system...

shown as a black dashed line and the SBDOT lesion shown as a white dashed line. The photocoagulation zones are clearly visible on the photographys and T2w images.

In Fig. 6, the temporal evolution of the coagulation zone size in the two tissues from the SBDOT reconstruction is compared with the $55^{\circ} \mathrm{C}$ MRI contours, whereas Table 3 summarizes the final lesion sizes. Note that the temperature starts to fall as soon as the treatment laser is turned off, whereas the reconstructed coagulation zone remains constant, as expected. In general, SBDOT systematically overestimates the lesion size compared with MRT in the earlier stages of treatment when the coagulation zones were small, albeit by $<1 \mathrm{~mm}$. However, the two monitoring methods came into close agreement as the coagulation zone expanded and as the coagulation boundary progressed toward the transrectal probe. This was confirmed also by the direct photographic measurements at the end of treatment.

The accuracy in the coagulation size derived from MRT is limited by the axial pixel resolution $\left(1 \times 1 \mathrm{~mm}^{2}\right)$ and uncertainty in the temperature assumed for coagulation: the transverse radius increases by up to $0.6 \mathrm{~mm}$ as coagulation thresholds were increased from $53^{\circ} \mathrm{C}$ to $57^{\circ} \mathrm{C}$. The accuracy of the TRDOT reconstructions was estimated by the following bootstrap procedure. For each measurement set, i.e., at each treatment time point, the reconstruction was repeated 50 times using random sets of the 64 source-detector pairs ${ }^{44}$ and the standard deviation
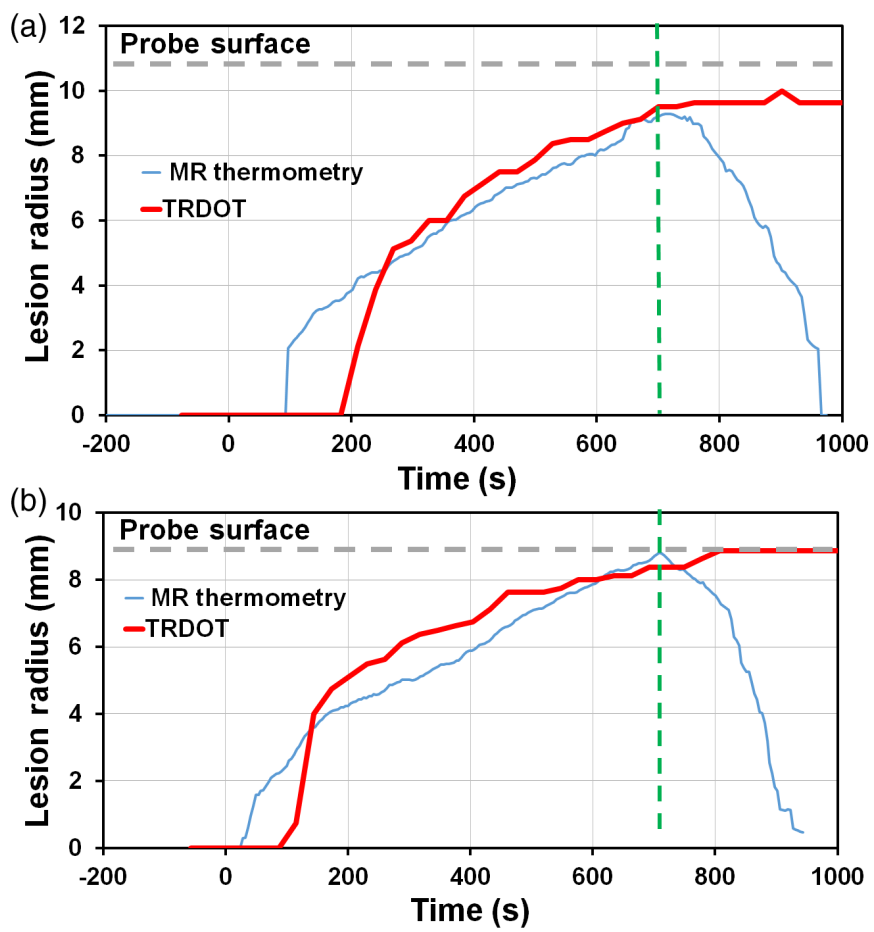

Fig. 6 Comparison in ex vivo (a) porcine and (b) bovine muscle of the coagulation radius given by the $55^{\circ} \mathrm{C}$ MRT contour and from the SBDOT reconstruction. PTT started at $t=0 \mathrm{~s}$ and stopped $t=700 \mathrm{~s}$ (dashed green line).

Table 3 PTT lesion radius $(\mathrm{mm})$ at the end of treatment from T2w MRI and MR thermometry (55 $\mathrm{C}$ threshold), TRDOT, and direct measurement from photographs.

\begin{tabular}{lcccccccc}
\hline \hline & & \multicolumn{3}{c}{ Transverse $(\mathrm{mm})$} & & \multicolumn{3}{c}{ Longitudinal $(\mathrm{mm})$} \\
\cline { 3 - 4 } Tissue & PTT fiber depth & TRDOT & MR T2w & MRT & & TRDOT & MR T2w & Photo \\
\hline Porcine & 10.7 & 9.6 & 7.3 & 9.1 & 12.0 & N/A & 12.5 \\
Bovine & 9.0 & 9.0 & 8.0 & 9.0 & & 11.0 & 10.9 & 12.5 \\
\hline \hline
\end{tabular}

Note: N/A, not available. 
$\mathrm{Li}$ et al.: Preclinical evaluation of a clinical prototype transrectal diffuse optical tomography system...

in the coagulation size was calculated. The overall uncertainty in the reconstructed coagulation zone radius was $\pm 0.6 \mathrm{~mm}$ transversely and $\pm 3 \mathrm{~mm}$ longitudinally. This could not be attributed to instrument noise, which was $<1 \%$ for all source-detector pairs, both at baseline and during PTT monitoring.

Since the TRDOT probe operates in reflectance geometry, it is less sensitive to optical changes occurring deep in the tissue. Consequently, early in the treatment, the changes in detected optical signal are small, resulting in an underestimate of the size of the coagulation zone. However, this is not of concern in this clinical application. If required, it could be addressed by placing interstitial source/detector fibers within the treatment zone but at the cost of added complexity and invasiveness. It is also possible that our light modeling method is suboptimal, especially at the boundary of the native and coagulated tissues. We are investigating the mesh resolution and assumption of diffusion theory by comparisons to Monte Carlo simulations.

Reconstruction of the final coagulation lesion size was generally insensitive to the initial estimates of the optical properties during reconstruction: varying the initial $\mu_{a}$ and $\mu_{s}^{\prime}$ values by a factor of 2 typically resulted in $<10 \%$ error in the reconstructed radius.

\subsection{In Vivo Study}

Table 4 summarizes the optical properties of native prostate tissue in vivo at $750 \mathrm{~nm}$ measured by the DR and the TRDOT probes, those of coagulated prostate measured by the TRDOT probe and, for comparison, literature values of ex vivo samples measured using a double integrating sphere method. ${ }^{45}$ The DR values were used as the initial parameters in the reconstruction algorithm. The $\mu_{a}$ values of native prostate measured by the two probes were lower than the published ex vivo canine prostate values at 1064 and $633 \mathrm{~nm}^{45}$ The absorption decreased by about $40 \%$ upon photocoagulation. For $\mu_{s}^{\prime}$ in native prostate, the DR measurement was almost twice the TRDOT value, likely due to the former measuring only anterior prostate and the latter having contributions from 2 to $3 \mathrm{~mm}$ of rectal wall and connective tissue in addition to the posterior prostate. $\mu_{s}^{\prime}$ measured by the TRDOT probe showed nearly twofold increase upon coagulation, which is comparable to the 1.4-fold and 1.6-fold increases reported in ex vivo prostate samples at 1064 and $633 \mathrm{~nm}$, respectively. ${ }^{45}$

The interpretation of the tissue response as "binary," namely coagulated versus unaltered native tissue, is clearly an oversimplification in vivo, since temperature-induced homeostatic changes in blood perfusion in the prostate and/or rectum ${ }^{46,47}$ will affect the optical measurements. Blood accumulation around the PTT fiber (Fig. 7) is a further potential confounding factor that would impact primarily the reconstructed absorption value. The SBDOT reconstructions gave coagulation zone radii between 6 and $12 \mathrm{~mm}$ across the 6 animals and converged consistently post-treatment. During PTT, however, the reconstruction is affected markedly also by heat-induced physiological changes within the DOT-interrogated region. In our previous preliminary in vivo canine studies, we reported large cyclical fluctuations in TRDOT signals during PTT $^{27}$ and these are apparent also in the examples shown in Fig. S4 in the Supplementary Material. However, the photocoagulation zone expands monotonically and smoothly, suggesting

Table 4 Reconstructed optical properties at $750 \mathrm{~nm}$ (mean \pm 1 standard deviation) for native and coagulated canine prostate tissue in vivo ( $n=6$ dogs). Measured values are compared to those measured using double integrating sphere measurement in Ref. 45 at 1064 and $633 \mathrm{~nm}$.

\begin{tabular}{lccccc}
\hline \hline & \multicolumn{2}{c}{ Native } & & \multicolumn{2}{c}{ Coagulated } \\
\cline { 2 - 3 } \cline { 5 - 6 } & $\mu_{a}\left(\mathrm{~mm}^{-1}\right)$ & $\mu_{s}^{\prime}\left(\mathrm{mm}^{-1}\right)$ & & $\mu_{a}\left(\mathrm{~mm}^{-1}\right)$ & $\mu_{s}^{\prime}\left(\mathrm{mm}^{-1}\right)$ \\
\hline $750 \mathrm{~nm}$ (DR probe) & $0.036 \pm 0.008$ & $1.2 \pm 0.3$ & & \\
$750 \mathrm{~nm}$ (TRDOT) & $0.034 \pm 0.01$ & $0.66 \pm 0.2$ & & $0.020 \pm 0.008$ & $1.1 \pm 0.3$ \\
$1064 \mathrm{~nm}^{45}$ & $0.027 \pm 0.003$ & $1.76 \pm 0.13$ & & $0.019 \pm 0.002$ & $2.44 \pm 0.14$ \\
$633 \mathrm{~nm}^{45}$ & $0.073 \pm 0.007$ & $2.25 \pm 0.05$ & & $0.061 \pm 0.006$ & $3.59 \pm 0.17$ \\
\hline \hline
\end{tabular}



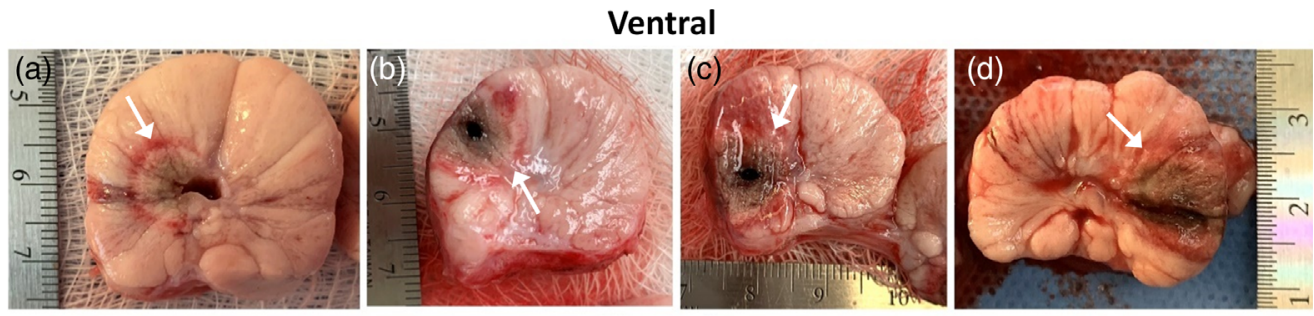

\section{Dorsal}

Fig. 7 Representative photographs of the photocoagulation zone (indicated by arrows) in four canine prostates excised shortly after completion of the treatment located (a) near the urethra, (b) upper left quandrant, (c) lower left quadrant, and (d) right side. The transrectal probe is located near the dorsal side.
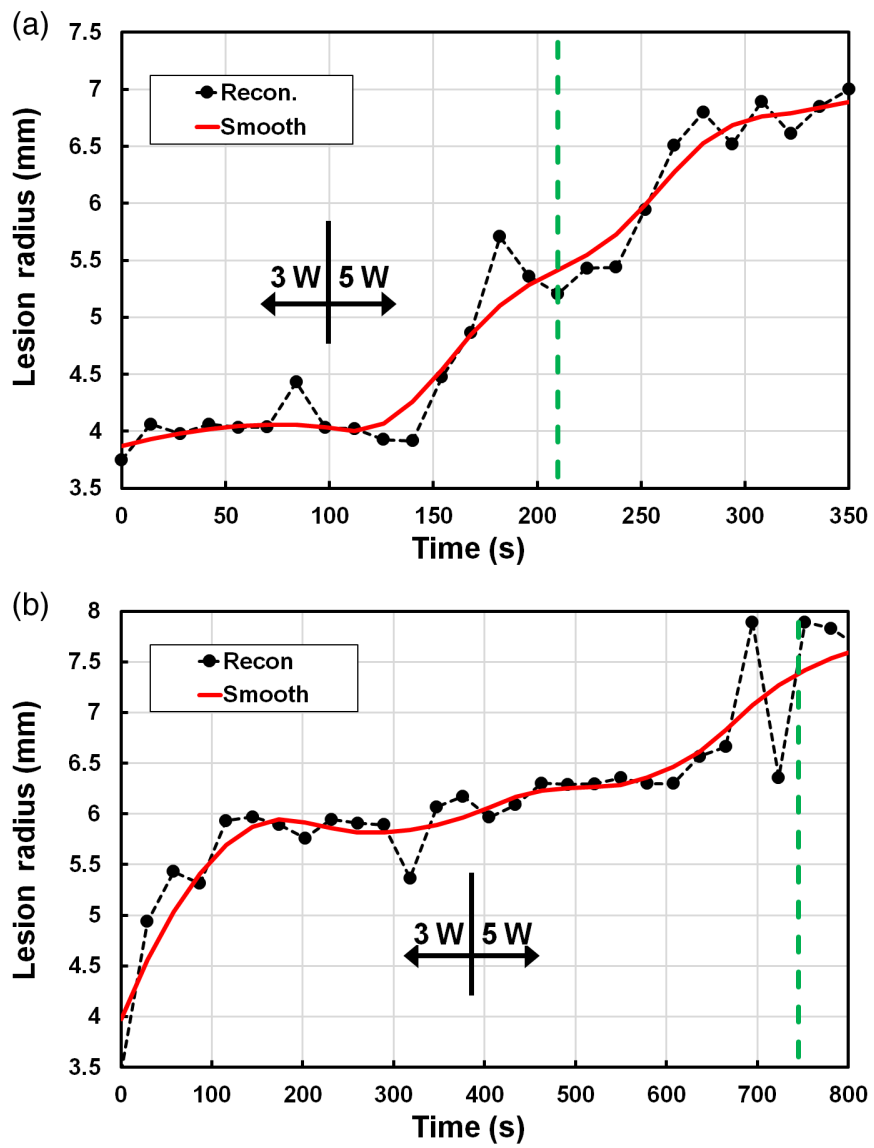

Fig. 8 Examples of SBDOT reconstructions during dual-power PTT in vivo with (a) four or (b) eight light sources activated with (red) and without (black) 5 -point smoothing. PTT starts at $t=0 \mathrm{~s}$ and stops at the green vertical line.

that this behavior is an artefact due to physiological responses to either the heating and/or mechanical stresses from probe placement: it remains to be seen if these fluctuations occur also in human patients during PTT. As shown in Fig. 8, applying five-point smoothing to the reconstructed axial radius largely eliminated the problem, allowing the true coagulation boundary growth to be monitored. This may also be improved further using multiple wavelengths in order to decouple the contribution of changes in blood flow from the true coagulative changes. An additional factor that adversely affects the accuracy of the coagulation monitoring is $28 \mathrm{~s}$ TRDOT scan time, during which the photocoagulation radius grows typically by $\sim 0.5 \mathrm{~mm}$, so that the system is not sampling the same coagulation zone between the start and end of each scan. 
Figure 8 presents two specific cases of in vivo PTT on canine prostate where the treatment power was increased during PTT. For the case shown in Fig. 8(a), TRDOT used four light sources and all eight detection channels. The post-smoothing reconstruction resulted in a final reconstructed radius of $6.8 \pm 0.2 \mathrm{~mm}$ compared to $7.0 \mathrm{~mm}$ by direct visualization after resection: here the treatment fiber and coagulation front were located at 10 and $3 \mathrm{~mm}$, respectively, from the TRDOT probe surface. Figure 8(b) shows an example using 8 light sources and 8 detection channels that yielded a final reconstructed radius of $7.7 \pm 0.2 \mathrm{~mm}$ compared to $8.0 \mathrm{~mm}$ directly with the PTT fiber and coagulation front at 11 and $3 \mathrm{~mm}$ from the TRDOT probe surface.

In both cases, when the treatment laser power was increased at the treatment midpoint, there was a corresponding increased rate of coagulation growth as measured by TRDOT, showing that TRDOT is properly tracking the dynamic changes, as required. We note that, even for closely matched dogs and using normal prostate, there is considerable animal-to-animal variation in the thermal response, which highlights the need for such monitoring in individual cases. Experiments to obtain point-by-point quantitative confirmation versus MR thermometry are planned and are expected to be successful since the final reconstructed size of the coagulation zone is consistent with direct measurements post mortem.

\section{Conclusions}

This work has confirmed the feasibility and accuracy of TRDOT with shape-based reconstruction of the coagulation zone for monitoring PTT treatments. This particular algorithm is not designed for reconstructing the tissue optical properties with high accuracy but, rather, to localize the photocoagulation boundary accurately with respect to the rectal wall. This is the most critical capability to guide treatment so as to achieve complete focal-lesion ablation while ensuring safety. The above experiments in phantoms, ex vivo tissues, and an in vivo canine prostate model have confirmed that TRDOT can achieve the target accuracy of $\pm 1 \mathrm{~mm}$. Caveats to this conclusion are limitations in the sensitivity and reconstruction accuracy when using our transrectal probe, where data are collected in the reflectance mode and the assumption that the coagulated zone is generally elliptical in shape. Our earlier simulations demonstrated that there were limits in reconstruction accuracy when assuming an elliptical shape in cases where irregularities in the true coagulation zone shape deviated from an elliptical shape by more than $2 \mathrm{~mm}^{29}$

Planned equipment updates aim to reduce the scan time to $\sim 5 \mathrm{~s}$ while also acquiring at multiple wavelengths. Additionally, in the present studies, reconstruction was performed retrospectively using a MATLAB finite-element model for light propagation on a standard PC, typically requiring several minutes. In clinical practice, reconstructions need to be near real time, for which we are exploring alternative reconstruction methods using look-up tables generated from forward Monte Carlo simulations as well as GPU accelerated hardware. ${ }^{48}$ We also plan to combine TRDOT with TRUS imaging that will provide high-resolution anatomical information to complement the functional information from TRDOT, both for improved tumor target localization and PTT treatment monitoring/control. Finally, we note that, although demonstrated here for PTT, TRDOT should be applicable to other thermal focal modalities as well as to treatments in other organs with appropriate DOT probe configurations.

\section{Disclosures}

The authors have no financial interests related to the material in this paper.

\section{Acknowledgments}

This work was supported by the Terry Fox Research Institute (Grant No. 1075). The authors would like to thank Dr. Michael Daly for performing CT scans in the phantom study, Dr. Warren Foltz for performing MRI procedures in the ex vivo study, and Dr. Alexander Gregor for assisting in the canine surgeries. 


\section{References}

1. A. W. Nelson and N. Shah, "Prostate cancer," Surgery 37(9), 500-507 (2019).

2. N. Patil and K. Gaitonde, "Clinical perspective of prostate cancer," Top. Magn. Reson. Imaging 25(3), 103-108 (2016).

3. M. Valerio et al., "The role of focal therapy in the management of localised prostate cancer: a systematic review," Eur. Urol. 66(4), 732-751 (2014).

4. R. P. Werntz and S. E. Eggener, "Novel focal therapy treatment options for prostate cancer," Curr. Opin. Urol. 28(2), 178-183 (2018).

5. A. Ouzzane et al., "Focal therapy as primary treatment for localized prostate cancer: definition, needs and future," Futur. Oncol. 13(8), 727-741 (2017).

6. E. J. Bass and H. U. Ahmed, "Focal therapy in prostate cancer: a review of seven common controversies," Cancer Treat. Rev. 51, 27-34 (2016).

7. M. Perera et al., "An update on focal therapy for prostate cancer," Nat. Rev. Urol. 13(11), 641-653 (2016).

8. K. J. Tay et al., "Surveillance after prostate focal therapy," World J. Urol. 37(3), 397-407 (2019).

9. S. N. Goldberg, G. S. Gazelle, and P. R. Mueller, "Thermal ablation therapy for focal malignancy: a unified approach to underlying principles, techniques, and diagnostic imaging guidance," Am. J. Roentgenol. 174(2), 323-331 (2000).

10. M. A. Ansari, M. Erfanzadeh, and E. Mohajerani, "Mechanisms of laser-tissue interaction: II. Tissue thermal properties," J. Lasers Med. Sci. 4(3), 99-106 (2013).

11. S. Puccini et al., "Simulations of thermal tissue coagulation and their value for the planning and monitoring of laser-induced interstitial thermotherapy (LITT)," Magn. Reson. Med. 49(2), 351-362 (2003).

12. J. Heisterkamp, R. Van Hillegersberg, and J. N. M. IJzermans, "Critical temperature and heating time for coagulation damage: implications for interstitial laser coagulation (ILC) of tumors," Lasers Surg. Med. 25(3), 257-262 (1999).

13. U. Lindner et al., "Focal laser ablation for prostate cancer followed by radical prostatectomy: validation of focal therapy and imaging accuracy," Eur. Urol. 57(6), 1111-1114 (2010).

14. U. Lindner et al., "Image guided photothermal focal therapy for localized prostate cancer: phase I trial," J. Urol. 182(4), 1371-1377 (2009).

15. O. Raz et al., "Real-time magnetic resonance imaging-guided focal laser therapy in patients with low-risk prostate cancer," Eur. Urol. 58(1), 173-177 (2010).

16. Y. Al-Hakeem et al., "Magnetic resonance image-guided focal laser ablation in clinically localized prostate cancer: safety and efficacy," ANZ J. Surg. 89(12), 1610-1614 (2019).

17. S. Natarajan et al., "Focal laser ablation of prostate cancer: phase I clinical trial," J. Urol. 196(1), 68-75 (2016).

18. S. E. Eggener et al., "Phase II evaluation of magnetic resonance imaging guided focal laser ablation of prostate cancer," J. Urol. 196(6), 1670-1675 (2016).

19. E. Knull et al., "Evaluation of tumor coverage after MR-guided prostate focal laser ablation therapy," Med. Phys. 46(2), 800-810 (2019).

20. Y. Hoshi and Y. Yamada, "Overview of diffuse optical tomography and its clinical applications," J. Biomed. Opt. 21(9), 091312 (2016).

21. V. Venugopal, Q. Fang, and X. Intes, "Multimodal diffuse optical imaging for biomedical applications," Biophoton. Med. Appl. 3-24 (2015).

22. G. Xu et al., "Trans-rectal ultrasound-coupled near-infrared optical tomography of the prostate Part I: Simulation,” Opt. Express 16(22), 17484-17504 (2008).

23. Z. Jiang et al., "Trans-rectal ultrasound-coupled near-infrared optical tomography of the prostate. Part II: experimental demonstration," Opt. Express 16(22), 17505-17520 (2008).

24. Z. Jiang et al., "In vivo trans-rectal ultrasound-coupled optical tomography of a transmissible venereal tumor model in the canine pelvic canal," J. Biomed. Opt. 14(3), 030506 (2009).

25. V. C. Kavuri and H. Liu, "Hierarchical clustering method to improve transrectal ultrasoundguided diffuse optical tomography for prostate cancer imaging," Acad. Radiol. 21(2), 250-262 (2014). 
$\mathrm{Li}$ et al.: Preclinical evaluation of a clinical prototype transrectal diffuse optical tomography system...

26. J. He et al., "Diffuse optical tomography to monitor the photocoagulation front during interstitial photothermal therapy: numerical simulations and measurements in tissue-simulating phantoms," Photonics Lasers Med. 3(3), 241-254 (2014).

27. C. L. Li et al., "Pre-clinical validation of transrectal diffuse optical tomography for monitoring photocoagulation progression during photothermal therapy of prostate cancer," Proc. SPIE 10871, 1087115 (2019).

28. J. He et al., "A clinical prototype transrectal diffuse optical tomography (TRDOT) system for in vivo monitoring of photothermal therapy (PTT) of focal prostate cancer," IEEE Trans. Biomed. Eng. 67(7), 2119-2129 (2020).

29. R. A. Weersink et al., "Shape-based reconstruction for transrectal diffuse optical tomography monitoring of photothermal focal therapy of prostate cancer: simulation studies," J. Biomed. Opt. 22(4), 045004 (2017).

30. C. M. MacLaughlin et al., "Porphysome nanoparticles for enhanced photothermal therapy in a patient-derived orthotopic pancreas xenograft cancer model: a pilot study," J. Biomed. Opt. 21(8), 084002 (2016).

31. T. J. Farrell, S. M. Patterson, and B. C. Wilson, "A diffusion theory model of spatially resolved, steady-state diffuse reflectance for the noninvasive determination of tissue optical properties in vivo," Med. Phys. 19(4), 879-888 (1992).

32. H. Dehghani et al., "Near infrared optical tomography using NIRFAST: algorithm for numerical model and image reconstruction," Commun. Numer. Methods Eng. 25(6), 711-732 (2009).

33. T. C. Zhu et al., "Optical properties of human prostate at $732 \mathrm{~nm}$ measured in vivo during motexafin lutetium-mediated photodynamic therapy," Photochem. Photobiol. 81(1), 96-105 (2005).

34. R. A. Weersink et al., "Techniques for delivery and monitoring of TOOKAD (WST09)mediated photodynamic therapy of the prostate: clinical experience and practicalities," J. Photochem. Photobiol. B Biol. 79, 211-222 (2005).

35. T. Svensson et al., "In vivo optical characterization of human prostate tissue using nearinfrared time-resolved spectroscopy," J. Biomed. Opt. 12(1), 014022 (2007).

36. J. L. Sandell and T. C. Zhu, "A review of in-vivo optical properties of human tissues and its impact on PDT," J. Biophotonics 4(11-12), 773-787 (2011).

37. V. K. Nagarajan and B. Yu, "Monitoring of tissue optical properties during thermal coagulation of ex vivo tissues," Lasers Surg. Med. 48(7), 686-694 (2016).

38. G. Marquez et al., "Anisotropy in the absorption and scattering spectra of chicken breast tissue," Appl. Opt. 37(4), 798-804 (1998).

39. S. Mosca et al., "Optical characterization of porcine tissues from various organs in the 650-1100 nm range using time-domain diffuse spectroscopy," Biomed. Opt. Express 11(3), 1697 (2020).

40. A. Kienle et al., "Spatially resolved absolute diffuse reflectance measurements for noninvasive determination of the optical scattering and absorption coefficients of biological tissue," Appl. Opt. 35(13), 2304-2314 (1996).

41. J. Xia et al., "Heating induced optical property changes in beef muscle," J. Food Eng. 84(1), 75-81 (2008).

42. S. Grabtchak, L. G. Montgomery, and W. M. Whelan, "Optical absorption and scattering properties of bulk porcine muscle phantoms from interstitial radiance measurements in 650-900 nm range," Phys. Med. Biol. 59(10), 2431-2444 (2014).

43. M. T. Adams et al., "Thermal dose dependent optical property changes of ex vivo chicken breast tissues between 500 and 1100 nm," Phys. Med. Biol. 59(13), 3249-3260 (2014).

44. B. Efron and R. Tibshirani, "Boostrap methods for standard errors, confidence intervals, and other measures of statistical accuracy," Stat. Sci. 1(1), 54-77 (1986).

45. W. H. Nau, R. J. Roselli, and D. F. Milam, "Measurement of thermal effects on the optical properties of prostate tissue at wavelengths of 1,064 and $633 \mathrm{~nm}$," Lasers Surg. Med. 24(1), 38-47 (1999).

46. B.-M. Kim et al., "Role of dynamic changes in blood perfusion and optical properties in thermal coagulation of the prostate," Proc. SPIE 2391, 443-450 (1995). 
47. S. Thomsen et al., "Temperatures associated with thermally-induced red blood cell changes in tissues irradiated in vivo," Proc. SPIE 2130, 156-163 (1994).

48. J. Cassidy et al., "High-performance, robustly verified Monte Carlo simulation with FullMonte," J. Biomed. Opt. 23(8), 085001 (2018).

Celina L. Li received her double BSc degrees in biology and physics from the University of British Columbia, Vancouver, Canada, in 2017 and her MSc degree in medical biophysics from the University of Toronto, Toronto, Canada, in 2020. She is a graduate student at Biophotonics@ Tyndall, IPIC, Tyndall National Institute, Ireland. Her research interest includes diffuse reflectance spectroscopy, machine learning, and translation research.

Carl J. Fisher received his BSc (Hons) degree in biochemistry from McGill University in 2009 and his $\mathrm{PhD}$ in medical biophysics from the University of Toronto in 2016. He is currently pursuing his degree in medicine at the University College Cork. He is a researcher in the Department of Biophotonics at Tyndall National Institute, Cork, Ireland. His research interests include integration of optics in surgical devices and use of biophotonics for therapeutic applications.

Brian C. Wilson is a professor in the Department of Medical Biophysics at the University of Toronto and a senior scientist at the Princess Margaret Cancer Centre. He directs a multidisciplinary research program developing optical- and nanotechnology-based techniques for medical diagnosis, treatment, and interventional guidance. He has developed and translated to the clinical several optical spectroscopy techniques and is the recipient of several national and international awards for his translational research.

Robert A. Weersink received his PhD in chemical physics from the University of Toronto in 1994. He is a clinical physicist in the Department of Radiation Medicine at the Princess Margaret Cancer Centre and an assistant professor in the Departments of Radiation Oncology, Medical Biophysics, and Biomedical Engineering at the University of Toronto. His research interests include image-guided therapies in surgery and radiation medicine with a focus on translational research. 